\title{
ASSOCIATION OF CANADIAN UNIVERSITY DEPARTMENTS OF ANESTHESIA POSTGRADUATE FELLOWSHIPS IN ANESTHESIA
}

\begin{tabular}{|c|c|c|c|}
\hline University & Position & Next Available & Contact \\
\hline $\begin{array}{l}\text { Dalhousie University } \\
\text { Halifax, Nova Scotia }\end{array}$ & $\begin{array}{l}\text { 2-Anesthesia Research } \\
\text { l-Chronic Pain Management } \\
\text { l-Acute Pain Management } \\
\text { l-Obstetric } \\
\text { l-Pediatric }\end{array}$ & $2000 / 2001$ & $\begin{array}{l}\text { Dr. T.J. Coonan, } \\
\text { Department of Anesthesia, QEII Health Sciences Centre, Victoria General Site } \\
1278 \text { Tower Road, Halifax, Nova Scotia B3H 2Y9, Phone: (902) 473-2331 }\end{array}$ \\
\hline $\begin{array}{l}\text { McGill University } \\
\text { Montréal, Québec }\end{array}$ & $\begin{array}{l}\text { 1-Cardiovascular } \\
\text { 2-Obstetric } \\
\text { 1-Pain Management } \\
\text { 2-Pediatric } \\
\text { 1-Neuroanesthesia } \\
\text { 1-Clinical/Research } \\
\text { 1-Thoracic }\end{array}$ & $2000 / 2001$ & $\begin{array}{l}\text { Dr. Francesco Carli, Chairman, } \\
\text { Department of Anesthesia, McGill University, } \\
\text { Royal University Hospital, } 687 \text { Pine Avenue West, Room F9.16, } \\
\text { Montreal, Quebec H3A 1Al, Phone: (514) 842-1231, Local } 5342\end{array}$ \\
\hline \multirow[t]{5}{*}{$\begin{array}{l}\text { University of Toronto } \\
\text { Toronto, Ontario }\end{array}$} & $\begin{array}{l}\text { 2-Thoracic (Clinical/Research) } \\
\text { 8-Cardiac \& Intensive Care } \\
\text { (ICU/Clinical/ Research) } \\
\text { 4-Pain (Acute/Chronic/Clinical } \\
\text { Research) } \\
\text { 3-Regional Anesthesia } \\
\quad \text { (Clinical/Research) } \\
\text { 2-Transplantation } \\
\text { (Heart/Lung/Liver) } \\
\text { 4-Neurosurgical } \\
\text { (Clinical/Research) } \\
\text { 2-Ambulatory Anesthesia } \\
\text { (Clinical/Research) } \\
\text { 4-Clinical Anesthesia } \\
\text { 4-Obstetrical } \\
\text { (Clinical/Research - } \\
\text { Mt. Sinai Hospital) }\end{array}$ & $2000 / 2001$ & $\begin{array}{l}\text { Dr. Alan N. Sandler, Anesthesiologist-in-Chief, Department of Anesthesia, } \\
\text { University Health Network and Mt. Sinai Hospital, } 585 \text { University Avenue, } \\
\text { Toronto, Ontario, Canada M5G 2C4 } \\
\text { Fax: (416) } 340-3698 \text {, Phone: (416) 340-3275, } \\
\text { E-mail: asandler@torhosp.toronto.ca }\end{array}$ \\
\hline & $\begin{array}{l}\text { 1-Obstetrical } \\
\quad(\text { Clinical/Research })\end{array}$ & $2000 / 2001$ & $\begin{array}{l}\text { Dr. S. Halpern, Director of Obstetrical Anesthesia, Department of Anaesthesia, } \\
\text { Women's College Hospital, } 76 \text { Grenville Street, } \\
\text { Toronto, Ontario, Canada M5S 1B2, Phone: (416) 323-6269 }\end{array}$ \\
\hline & $\begin{array}{l}\text { 1-Clinical/Research } \\
\text { 8-Pediatric/Anesthesia } \\
\text { (Clinical/Research/Pain } \\
\text { management) }\end{array}$ & $\begin{array}{l}\text { Jan or July } \\
2000 \text { for l yr } \\
2000 / 2001\end{array}$ & $\begin{array}{l}\text { Dr. Jean Kronberg, Anesthetist-in-Chief, Women's College Hospital, } \\
76 \text { Grenville St., Toronto, Ontario, M5S 1B2, Phone: (416) 323-6008 } \\
\text { Dr. L. Roy, Anesthetist-in-Chief, Department of Anesthesia, Hospital } \\
\text { for Sick Children, } 555 \text { University Avenue, Toronto, Ontario, Canada M5G 1X8 } \\
\text { Phone: (416) 813-7445, Fax: (416) 813-7543, E-mail: roy@anes.sickkids.on.ca } \\
\text { Information available at: http://wwwsickkids.on.ca/anesthesia }\end{array}$ \\
\hline & $\begin{array}{l}\text { 8-Pediatric Critical Care } \\
\quad \text { (Clinical) } \\
\text { 2-Pediatric Critical Care } \\
\quad \text { (Research) }\end{array}$ & $2000 / 2001$ & $\begin{array}{l}\text { Dr. G.A. Barker, Chief, Department of Critical Care Medicine, } \\
\text { Hospital for Sick Children, } 555 \text { University Avenue, Toronto, } \\
\text { Ontario, Canada M5G 1X8, Phone: (416) } 813-6860\end{array}$ \\
\hline & $\begin{array}{l}\text { 1-Trauma/Critical Care } \\
\text { (Clinical/Research) } \\
\text { 3-Clinical/Research Anesthesia } \\
\text { 1-Critical Care (Clinical/Research) } \\
\text { 1-Cardiac (Clinical/Research) } \\
\text { 1-Regional Anesthesia } \\
\text { (Clinical/Research) } \\
\text { 6-Pain/Critical Care/Trauma/ } \\
\text { Cardiac (including echocardio- } \\
\text { graphy)/Neuro or customized } \\
\text { program (Clinical Research) }\end{array}$ & $2000 / 2001$ & $\begin{array}{l}\text { Dr. J.H. Devitt, Anesthetist-in-Chief, Department of Anesthesia, Sunnybrook } \\
\text { Health Sciences Centre, } 2075 \text { Bayview Avenue, M3-200, Toronto, Ontario } \\
\text { M4N 3M5, Phone: (416) 480-4864, Fax: (416) 480-6039, } \\
\text { E-mail: hugh.devitt@sunnybrook.on.ca } \\
\text { Dr. D.K. Rose, Anesthetist-in-Chief, Department of Anesthesia, } \\
\text { St. Michael's Hospital, 30 Bond Street, Toronto, Ontario, Canada, M5B 1W8 } \\
\text { Phone: (416) 864-5071, Fax: (416) 864-6014, E-mail: rosek@smh.toronto.on.ca } \\
\text { More detailed information on these fellowship positions may be obtained from the } \\
\text { Department of Anaesthesia home page on the WWW at } \\
\text { http://www.utoronto.ca/anaesthesia }\end{array}$ \\
\hline \multirow[t]{3}{*}{$\begin{array}{l}\text { University of Ottawa, } \\
\text { Ottawa, Ontario }\end{array}$} & $\begin{array}{l}\text { l-Chronic Pain Management } \\
\text { l-Obstetrical Anesthesia } \\
\text { l-Regional Anesthesia } \\
\text { l-Cardiac Anesthesia }\end{array}$ & $\begin{array}{l}\text { January } 2000 \\
\text { January } 2000 \\
\text { July } 2000\end{array}$ & $\begin{array}{l}\text { Dr. Dennis Reid, Head, Department of Anesthesia, Ottawa Hospital - Alta Vista } \\
\text { Site, } 501 \text { Smyth Road, Ottawa, Ontario KlH 8L6, Phone: (613) 737-8187 } \\
\text { Dr. J. Robblee, Head, University of Ottawa Heart Institute, } \\
40 \text { Ruskin, Ottawa. Phone: }(613) 761-4379\end{array}$ \\
\hline & $\begin{array}{l}\text { 1-Obstetric Anesthesia } \\
\text { l-Chronic Pain } \\
\text { l-Vascular Anesthesia } \\
\text { l-Thoracic }\end{array}$ & & $\begin{array}{l}\text { Dr. Larry Byford, Chief, Department of Anesthesia, B30, Ottawa Hospital-Civic } \\
\text { Site, } 1053 \text { Carling Avenue, Ottawa, Ontario KlY 4E9, Phone: (613) } 761-4940\end{array}$ \\
\hline & 2-Pediatric Anesthesia & January 2000 & $\begin{array}{l}\text { Dr. William Splinter, Chief, Department of Anesthesia, Children's Hospital of } \\
\text { Eastern Ontario, } 401 \text { Smyth Road, Ottawa, Ontario, Phone: (613) 737-2431 }\end{array}$ \\
\hline $\begin{array}{l}\text { University of Calgary } \\
\text { Calgary, Alberta }\end{array}$ & l-Pediatric & $2000-2001$ & $\begin{array}{l}\text { Dr. G.V. Goresky, Acting Regional Clinical Department Head, Department of } \\
\text { Anesthesia, Foothills Hospital, The University of Calgary, 1403-29th St. N.W., } \\
\text { Calgary, Alberta T2N 2T9 Phone: }(403) 670-1430\end{array}$ \\
\hline
\end{tabular}




\section{ASSOCIATION OF CANADIAN UNIVERSITY DEPARTMENTS OF ANESTHESIA POSTGRADUATE FELLOWSHIPS IN ANESTHESIA}

\begin{tabular}{|c|c|c|c|}
\hline University & Position & Next Available & Contact \\
\hline \multirow[t]{2}{*}{$\begin{array}{l}\text { McMaster University } \\
\text { Hamilton, Ontario }\end{array}$} & $\begin{array}{l}\text { Hamilton Health } \\
\text { Sciences Corporation: } \\
\text { 1-Epidemiology/Anesthesia } \\
\quad \text { (application should be received } \\
\quad \text { by Nov. 6th, 1999) } \\
\text { 2-Information/Anesthesia } \\
\text { 3-Pain } \\
\text { 4-Cardiac Anesthesia } \\
\text { 5-Pediatric Anesthesia } \\
\text { St. Joseph's Hospital: } \\
\text { 1-Clinical/Research } \\
\text { 2-Thoracic Anaesthesia }\end{array}$ & $2000 / 2001$ & $\begin{array}{l}\text { Dr. H. Yang, Chairman, Department of Anesthesia, McMaster University } \\
\text { Rm.2U1, } 1200 \text { Main Street West, Hamilton, Ontario L8N 3Z5, } \\
\text { Phone: (905) 521-2100 ext. 5166, Fax: (905) 523-1224 }\end{array}$ \\
\hline & $\begin{array}{l}\text { Hamilton Health Sciences } \\
\text { Corporation: } \\
\text { l-Critical Care }\end{array}$ & $2000 / 2001$ & $\begin{array}{l}\text { Dr. F. Baxter, Programme Director, Department of Critical Care, } \\
\text { Hamilton General Hospital, } 237 \text { Barton Street East, Hamilton, Ontario L } 8 \text { L } 2 \text { X2 } \\
\text { Phone: (905) 528-5565, Fax: (905) 577-1466 }\end{array}$ \\
\hline \multirow[t]{2}{*}{$\begin{array}{l}\text { The University of } \\
\text { Western Ontario } \\
\text { London, Ontario }\end{array}$} & $\begin{array}{l}\text { 6-Clinical/Research } \\
\text { (Ambulatory, Cardiac, Neuro, } \\
\text { Transplant, Pain Management, } \\
\text { Vascular, Thoracic Obstetrical \& } \\
\text { Regional Anesthesia \& Medical } \\
\text { Informatics. Individually or in } \\
\text { combination) } \\
\text { l-Research } \\
\text { (W.E. Spoerel Research } \\
\text { Fellowship) }\end{array}$ & $2000 / 2001$ & $\begin{array}{l}\text { Dr. K. Mary Lampe, Fellowship Coordinator, Department of Anesthesia, UWO } \\
\text { London Health Sciences Centre, University Campus, Office 2AN17, } \\
339 \text { Windermere Road, London, Ontario N6A 5A5, Phone (519) 663-3270 }\end{array}$ \\
\hline & $\begin{array}{l}\text { 4-Ministry of Health (Ontario) } \\
\text { Critical Care } \\
\text { 2-External Funds Clinical Fellows }\end{array}$ & $\begin{array}{l}2000 / 2001 \\
2000 / 2001\end{array}$ & $\begin{array}{l}\text { Dr. David Leasa, Postgraduate Medical Education Director, } \\
\text { London Health Sciences Centre, University Campus, } 339 \text { Windermere Road, } \\
\text { London, Ontario N6A 5A5, Phone: (519)663-3921; Fax: (519)663-8813 }\end{array}$ \\
\hline $\begin{array}{l}\text { The University of } \\
\text { British Columbia } \\
\text { Vancouver, } \\
\text { British Columbia }\end{array}$ & $\begin{array}{l}\text { Vancouver General Hospital } \\
2 / 3 \text {-Clinical/Research } \\
\text { B.C. Children's Hospital } \\
\text { 1/2-Clinical/Research } \\
\text { Women's Hospital } \\
\text { 1-Clinical/Research } \\
\text { St. Paul's Hospital } \\
\text { 1-Clinical Research }\end{array}$ & $2000 / 2001$ & $\begin{array}{l}\text { Dr. David Bevan, Professor \& Head, Department of Anesthesia, } \\
\text { University of British Columbia, } 910 \text { West 10th Avenue, Room 3200, } \\
\text { Vancouver, B.C. V5Z 4E3, Phone: (604) 875-4575; Fax: (604) 875-5344 }\end{array}$ \\
\hline $\begin{array}{l}\text { University of Alberta } \\
\text { Edmonton, Alberta }\end{array}$ & $\begin{array}{l}\text { 6-Cardiac, Pediatric, } \\
\text { Acute/Chronic Pain, Liver } \\
\text { Transplantation, Regional } \\
\text { Anesthesia }\end{array}$ & $2000 / 2001$ & $\begin{array}{l}\text { The Chairman, Department of Anesthesiology and Pain Medicine, } \\
\text { University of Alberta Hospital, } 8440-112 \text { Street, Room 3B2.32, } \\
\text { Edmonton, Alberta T6G 2B7, Phone: (780) 407-8861 }\end{array}$ \\
\hline $\begin{array}{l}\text { University of Manitoba } \\
\text { Winnipeg, Manitoba }\end{array}$ & 1-Obstetric Anesthesia & July 2000 & $\begin{array}{l}\text { Dr. Laurence Brownell, Program Director, Department of Anesthesia, } \\
60 \text { Pearl Street, Room LB } 315 \text {, Winnipeg, Manitoba R3E } 1 \text { X2 } \\
\text { E-mail: Ibrownell@hsc.mb.ca }\end{array}$ \\
\hline $\begin{array}{l}\text { Queen's University } \\
\text { Kingston, Ontario }\end{array}$ & $\begin{array}{l}\text { 2-Clinical/Research } \\
\text { Areas of interest: } \\
\text { 1. Vascular } \\
\text { 2. Cardiac } \\
\text { 3. Acute/chronic pain } \\
\text { 4. Ambulatory } \\
\text { 5. General }\end{array}$ & $2000 / 2001$ & $\begin{array}{l}\text { Dr. E.S. Shelley, Fellowship Coordinator, Department of Anesthesiology, } \\
\text { Queen's University, Kingston General Hospital, } 76 \text { Stuart Street } \\
\text { Kingston, Ontario K7L 2V7, Phone (613) 548-7827 }\end{array}$ \\
\hline $\begin{array}{l}\text { Université de Montréal, } \\
\text { Montréal, Québec }\end{array}$ & $\begin{array}{l}\text { 1-Pediatrics/Obstetrics (Clinical) } \\
\text { 1-Clinical Pharmacology } \\
\text { (Clinical/Research) } \\
\text { 1-Cardiovascular (Clinical/Research) } \\
\text { 1-Transoesophageal } \\
\text { Echocardiography (Clinical) } \\
\text { 1-Regional (Clinical) } \\
\text { 1-Acute and/or Chronic Pain } \\
\text { (Clinical) }\end{array}$ & $2000 / 2001$ & $\begin{array}{l}\text { Dr. Jean François Hardy, Chairman, } \\
\text { Département d'anesthésie, C.P. 6128, succursale A, } \\
\text { Montréal, Québec H3C 3J7, Phone: (514) 343-6466 }\end{array}$ \\
\hline
\end{tabular}

Footnotes 1. Qualifications: FRCPC or eligibility for examinations, except Vancouver General Hospital (completed minimum 3 years in Resident programme) and University of Toronto Grant Dependent Positions (minimum 2 years in Resident programme).

2. Remuneration, Medical Licencing Requirements , and other conditions: Contact individual departments.

3. Fellowships available for Canadian Citizens or Landed Immigrants.

4. Two-year programme; possible one-year overlap with base programme of anaesthesia. Required: FRCPC or eligibility for exams by end of programme. (List compiled June 1999 by Dr. David R. Bevan, Editor-in-Chief, Canadian Journal of Anesthesia, University of British Columbia, Vancouver, British Columbia) 